\title{
Decomposing Apricots: Tracking Transformation of Arsenic Sulfide pigments in a Dutch Golden Age Still-life.
}

\author{
Katrien Keune ${ }^{1}$, Jennifer Mass ${ }^{2}$, Florian Meirer ${ }^{3}$, Carol Pottash ${ }^{4}$, Annelies van Loon ${ }^{1,4}$, Alyssa Hull ${ }^{5}$, \\ and Apurva Mehta ${ }^{6}$ \\ 1. Faculty of Science, University of Amsterdam, 1090 GD Amsterdam, The Netherlands \\ 2. Scientific Research and Analysis Laboratory, Conservation Department, Winterthur Museum, \\ Winterthur, Delaware, USA \\ 3. Inorganic Chemistry and Catalysis, Debye Institute for Nanomaterials Science, Utrecht University, \\ Universiteitsweg 99, 3584 CG Utrecht, The Netherlands. \\ 4. Royal Picture Gallery Mauritshuis, The Hague, the Netherlands \\ 5. University of Delaware, Department of Chemistry, Newark, Delaware, USA \\ 6. Stanford Synchrotron radiation Lightsource, SLAC National Accelerator Laboratory, 2575 Sand Hill \\ Rd., Menlo Park, California, USA
}

Arsenic sulfide based yellow and red/orange pigments, orpiment $\left(\mathrm{As}_{2} \mathrm{~S}_{3}\right)$ and realgar $\left(\mathrm{As}_{4} \mathrm{~S}_{4}\right)$, have been used since antiquity. Though absent in Europe in middle-ages, these pigments came back from Asia Minor via Venice in the $15^{\text {th }}$ century. They were frequently used to paint furniture and historic interiors, often in place of gold. They were less common in easel paintings, but towards the end of the $17^{\text {th }}$ century they made a comeback, particularly in still-lifes. The late $18^{\text {th }}$ century saw gradual replacement of the Arsenic pigments by new pigments created by the revolution in chemistry.

Arsenic sulfide pigments are known to shift color under exposure to light. Realgar undergoes photoinduced polymorphism and turns bright yellow (to para-realgar) to colorless, whereas orpiment photooxidizes and becomes colorless. In here, we report on this transformation and characterization of the reaction products, based on investigation of two very different works of art from early $18^{\text {th }}$ century: Still life with five apricots by the Dutch master Adriaen Coorte (1704) (Royal Picture Gallery Mauritshuis, The Hague, The Netherlands) and an early American polychromed chest on stand attributed to Charles Guillam painted in Saybrook, Connecticut between 1710-1727 (Winterthur Museum, Wintherthur, USA). The small oil painting is painted on a thick yellow earth pigmented ground layer on canvas. Realgar, mixed with organic lake on gypsum substrate, is used in the paint build up of the apricots, with the highlights painted in vermilion. The apricots show signs of degradation. The paint layer is very crumbly, especially along the cracks, and appears to be vulnerable to further degradation when exposed to moisture and solvents. The Saybrook chest is painted in distemper, a proteinaceous binding medium, with foliate, crown and thistle, and fleur de lis motifs. The regions that were once painted with arsenic sulfide now have a mottled appearance of ivory and brown.

We used spectromicroscopy at an X-ray microprobe at Stanford Synchrotron Radiation Lightsource, supplemented by optical, infrared, and backscatter electron microscopy, Raman microspectroscopy. In here, we will focus on the X-ray results : X-ray fluorescence mode (XRF scanning) allowed precise mapping of local elemental distribution, while X-ray Near-Edge Absorption Structure (XANES) in combination with multi-energy XRF mode allowed identification and mapping of the gross chemical speciation. Cross-sectioned samples for these measurements were mounted in polyester resin blocks, and dry hand-polished. 


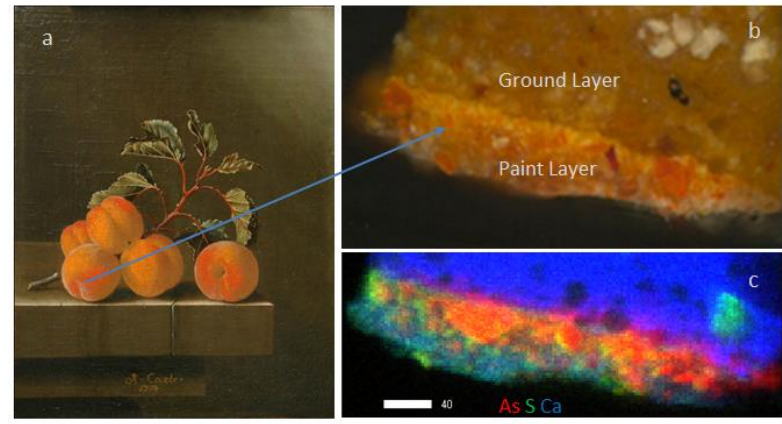

Figure 1: a) Adriaen Coorte, Still Life with Five Apricots, Mauritshuis; b) Paint cross-section from the region indicated, c) Composite elemental map - red $=$ As, green $=\mathrm{S}$, and blue $=\mathrm{Ca}$.

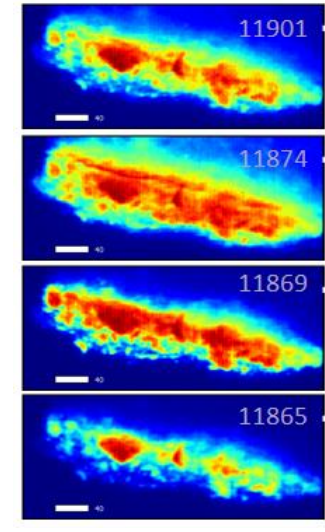

Figure2: As maps at $11865,11869,11874$, and $11901 \mathrm{eV}$

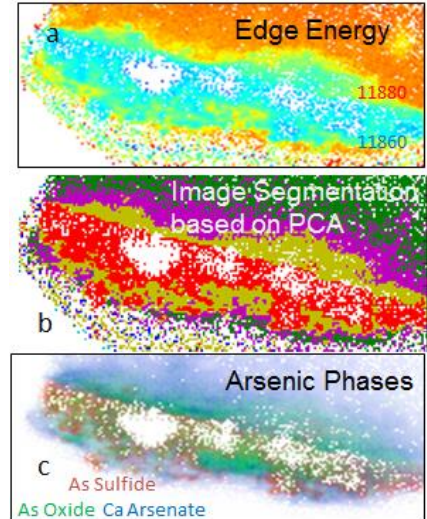

Figure 3: a) As $\mathrm{K}$ edge position; b) distribution of the principal components; c) As phase map

Three sets of X-ray Fluorscence (XRF) maps were collected with 2 micron resolution (see Figure 1b and Figure 2). The first set was collected at $12000 \mathrm{eV}, \sim 150 \mathrm{eV}$ above the $\mathrm{K}$ edge of As at $11868 \mathrm{eV}$. A second XRF map was collected below the As $\mathrm{K}$ edge (at $11820 \mathrm{eV}$ ) and with re-optimized detector geometry to obtain better $\mathrm{Ca}$ and $\mathrm{S}$ distribution. These two maps were combined to create a composite elemental distribution map (shown in Figure 1c). The composite map shows a very heterogeneous paint layer. The upper part of the layer is rich in $\mathrm{Ca}$ (as well as an area in the ground). A final set of XRF maps were collected by varying the X-ray incidence energy over the most species sensitive region of the As K edge. In total 20 such maps were collected, with 10 of them separated by $1 \mathrm{eV}$ from 11867 to 11877. These maps, four of them are shown in Figure 2, clearly indicate the presence of multiple As species. The distribution of the As K half edge position, shown in Figure 3a, where blue pixels indicate more reduced and red pixels more oxidized As, show two clear oxidation fronts; one between the pigment and the ground layer, and the second in the upper part of the paint layer. Image segmentation based clustering in Principal Component (PC) space shows layered morphology of the As species. (see Figure 3b) Based on Figure 3a and b, high-resolution XANES spectra covering $40 \mathrm{eVs}$ below and 300 $\mathrm{eV}$ above the As $\mathrm{K}$ edge with $0.25 \mathrm{eV}$ step size over the critical "white-line" region were collected at 77 locations on the sample. PC analysis of these XANES spectra indicates that there must be at least 3 and perhaps as many as 5 As components present. Comparison of the XANES spectra and their prinicipal components with published spectra and As reference compounds were carried out. These analysis suggest $\mathrm{As}_{2} \mathrm{~S}_{3}$ (most reduced), $\mathrm{Ca}_{3}\left(\mathrm{AsO}_{4}\right)_{2}$ (most oxidized), and $\mathrm{As}_{2} \mathrm{O}_{3}$ can adequately represents majority of the As speciation in the sample (see Figure 3c). The Saybrook cross-section shows that almost all the As in the paint layer has been oxidized to $\mathrm{Pb}_{3}\left(\mathrm{AsO}_{4}\right)_{2}$.

Arsenic speciation in the Coorte cross-section suggests formation of at least two (colorless) transformation products: $\mathrm{Ca}_{3}\left(\mathrm{AsO}_{4}\right)_{2}$ and $\mathrm{As}_{2} \mathrm{O}_{3}$. These products are very mobile. $\mathrm{As}_{2} \mathrm{O}_{3}$ has accumulated at the interface between paint and ground layer, whereas. $\mathrm{Ca}_{3}\left(\mathrm{AsO}_{4}\right)_{2}$ is found in the upper part of the paint layer, as well as in the ground. The arsenate species seems to have reacted with the gypsum $\left(\mathrm{CaSO}_{4}\right)$ in the paint layer and the chalk $\left(\mathrm{CaCO}_{3}\right)$ in the ground layer. $\mathrm{Tt}$ is yet unclear whether arsenates are formed directly from the photo-oxidatized arsenic sulfide or with a two stage oxidation, with $\mathrm{As}_{2} \mathrm{O}_{3}$ as an intermediate phase These results will be elaborated, supplemented with results from other complementary probes, and further discussed. 